\title{
PowerCool: Simulation of Integrated Microfluidic Power Generation in Bright Silicon MPSoCs
}

\author{
Arvind Sridhar ${ }^{1,2}$ Mohamed M. Sabry ${ }^{1}$ Patrick Ruch $^{2}$ David Atienza ${ }^{1}$ Bruno Michel $^{2}$ \\ ${ }^{1}$ Embedded Systems Lab (ESL), EPFL, Lausanne; ${ }^{2}$ IBM Research - Zurich \\ \{mohamed.sabry,david.atienza\}@epfl.ch; \{rvi,ruc,bmi\}@zurich.ibm.com
}

\begin{abstract}
Integrated microfluidic power generation and power delivery promises to be a disruptive packaging technology with the potential to combat dark silicon. It essentially consists of integrated microchannel-based electrochemical "flow cells" in a 2D/3D multiprocessor system-on-chip (MPSoC), that generate electricity to power up the entire or part of the chip, while also simultaneously acting as a high-efficiency microfluidic heat sink. Further development of this technology requires efficient modeling tools that would assess the efficacy of such solutions and help perform early-stage design space exploration. In this paper, we propose a compact mathematical model, called PowerCool, that performs electro-chemical modeling and simulation of integrated microfluidic power generation in MPSoCs. The accuracy of the model has been validated against fine-grained multiphysics simulations of flow cells in the COMSOL software that is unsuitable for EDA because of large simulation times. PowerCool model is demonstrated to be up to $425 \mathrm{x}$ times faster than COMSOL simulations while incurring a worst-case error of only 5\%. Furthermore, the PowerCool model has been used to study and assess the efficacy of this technology for a test MPSoC.
\end{abstract}

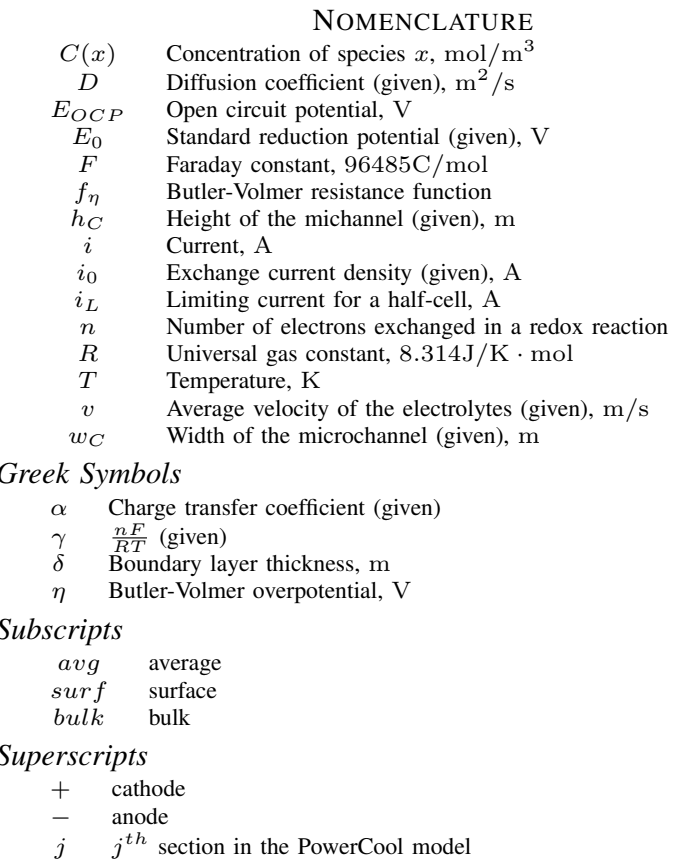

A. Sridhar and M. Sabry are authors of equal contributions to this work. This work has been partially supported by the Nano-Tera YINS RTD project (no. 20NA21_150939)financed by the Swiss Confederation and scientifically evaluated by SNSF, the EC FP7 GreenDataNet STREP project (Agreement No. 609000), and SNF Sinergia project REPCOOL (grant no. 147661). We would like to thank Sani Nassif for his inputs on the target architecture and power figures and the discussions on the power delivery network.

\section{INTRODUCTION}

The stagnation of Dennard scaling caused a shift from singleto multi-core processors and multi-processor system-on-chips (MPSoCs) in the mid-2000s. Now, it has started to limit even the scaling of such multi-core systems resulting in an under-utilization of MPSoCs. This under-utilization manifests itself in MPSoCs not functioning at their peak switching frequencies and also in the transient switching-off of many parts of the chip during operation. This phenomenon, called dark silicon [1], is the direct result of reduced energy efficiency in current devices.

Reduced energy efficiency is caused by multiple factors. The electronic packaging research community has responded with different solutions to each of them:

1. Increased energy is required for communication (vis a vis computation) between devices in ever-increasing chip sizes. 3D integration of silicon chips with reduced wire-lengths [2] has gained interest in the last five years in order to combat this trend.

2. Losses in power delivery at various levels of distribution network interconnects: PCB, package and on-chip. Also, increased chip area and performance must be accompanied with larger number of pins supplying power to the IC, and hence competing with communication lines for resources and undermining offchip bandwidths of MPSoCs. Supplying high-voltage power from the board and performing on-chip power conversion close to the devices using switching regulators can partially address this issue while also providing better control of voltage levels supplied to individual blocks of the IC [3].

3. Increased heat generated per unit foot-print area of the IC has risen alarmingly over the last two decades and threatens to compound even further with vertical 3D integration of high-performance processor cores. This puts tremendous pressure on existing cooling technologies and increases energy consumption required to cool devices to safe operating temperatures. Integrated liquid-cooling in 2D/3D ICs using silicon microchannels carrying single- or two-phase fluids have been demonstrated to be a highly-energy efficient solution to this problem [4].

All the solutions proposed so far only address specific aspects of energy-efficiency in MPSoCs. A study of the computational efficiency of existing electronic systems when compared with biological systems, as shown in Fig. 1, reveals the huge untapped potential to increase energy efficiency in MPSoCs. [6] proposes that disruptive technologies can have the potential to close this "efficiency gap" and combat dark silicon. Recently, a potentially disruptive technology was described by the authors of [7], called integrated microfluidic power generation and cooling, and is illustrated in Fig. 2. It provides a new paradigm of electronic design by using the same physical medium to power-up and cool-down 2D- or 3D-MPSoCs, via integrated silicon microchannels. In particular, an electrochemical species pair (a fuel and an oxidant) is injected into these microchannels. When conductive electrodes are connected to either side of these microchannels, these electrolytes undergo electrochemical oxidation-reduction, start to exchange ions with each other and electrons with the electrodes, 


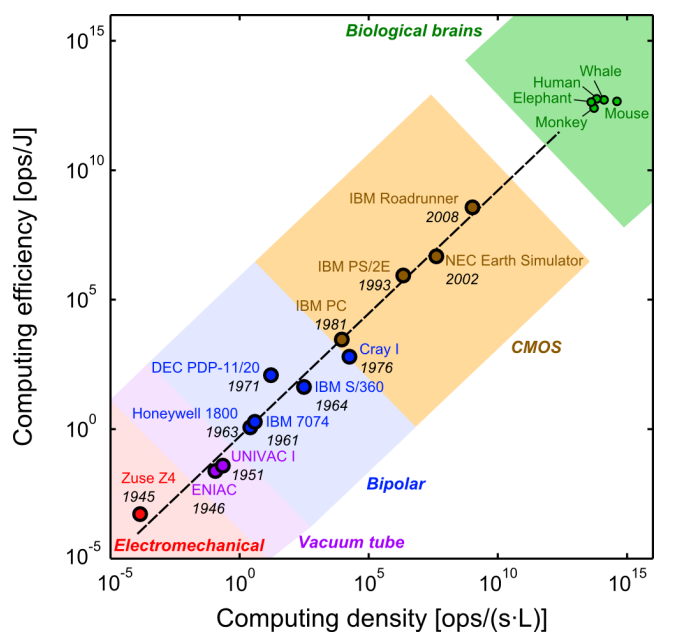

Fig. 1. Trends showing the increasing of computational density and energy efficiency in electronic systems vis-a-vis biological systems [5].

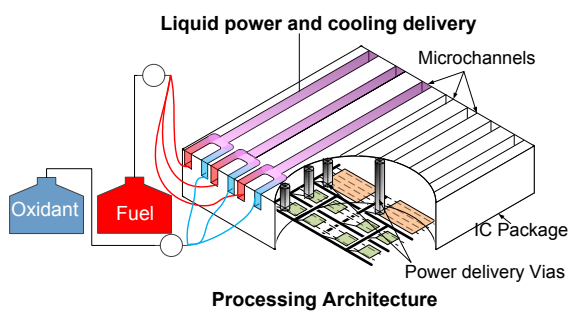

Fig. 2. Schematic diagram of the concept integrated microfluidic power supply and cooling of MPSoCs [7].

generating electric power as they flow along the microchannels. These flowing electrolytes also provide a means to heat-removal from underlying MPSoC via the high-efficiency microchannel heat sink. In summary, this integrated microfluidic power generation and cooling technology has three major consequences for the MPSoCs:

1. The MPSoC can be powered up fully or partially using these microchannel "redox flow cells" from within the package freeing up off-chip interconnects previously used for power delivery.

2. On-chip power supplies such as these significantly reduce losses.

3. Cooling of MPSoCs below safe operating temperatures can be simultaneously obtained from these flow cells, that have high efficiencies comparable to existing microchannel heat sinks.

Hence, all the three aspects of the "power wall" in dark silicon are potentially addressed in this solution enabling the powering up of bright silicon MPSoCs. The proposal of this new technology has also raised several questions: Is it capable of powering up an entire MPSoC? What are the I-V characteristics of such a power supply? How feasible is the integration of such a power supply with existing processes? Can the chips temperatures be maintained below safe limits of operation? What is the over-all energy efficiency of the combined power-supply and cooling? These questions must be answered and optimal design choices must be made at the early-stages of design for the further development and adoption of this technology in electronic systems. At the heart of this is the need for an efficient mathematical model than can simulate the electro-chemical and the thermal characteristics of the integrated microchannel flow cells and flow cell arrays.

In this paper, we propose a compact mathematical model, called PowerCool, that simulates electrochemical and thermal behavior of the integrated microfluidic power generation in MPSoCs to address the above need. Specifically, the following are the contributions of this work:

1. PowerCool: a compact mathematical model for the simulation of the electrochemical and thermal behaviour of integrated microchannel redox flow cell arrays.

2. The accuracy of the PowerCool model has been validated against fine-grained multiphysics simulations of flow cells in the COMSOL tool. Furthermore, the simulation times of the two models are compared. It is demonstrated that PowerCool is up to $425 \mathrm{x}$ times faster (380x on average) than COMSOL while incurring only $3.8 \%$ error (maximum $5 \%$ ) with respect to it.

3. As a demonstration of the use of the proposed model, PowerCool has been applied to a concept MPSoC that uses integrated microfluidic power generation and cooling. Combined electrochemical and thermal simulations are performed to assess the capacity of the redox flow cell array in powering up and cooling down the chip. In addition, the effect of chip temperatures on the electrochemistry of the redox flow cells is evaluated. It was found from simulations that the state-of-the-art redox flow cells can power-up the entire on-chip memory architecture of an existing MPSoC, while managing to cool-down the whole MPSoC. In addition, we find that increasing the temperature of the flowing fluids, either by increased inlet temperature or due to the increased natural heat dissipation of the chip, results in an increased generated power. Specifically, we have observed an increase of $23 \%$ of the generated power in our example when the inlet flow temperature is increased from $27^{\circ} \mathrm{C}$ to $37^{\circ} \mathrm{C}$. This observation has significant implications for early-stage design and optimization of such devices.

The rest of the paper is organized as follows: Section II briefly reviews the existing modeling approaches to microchannel redox flow cells. Section III describes the target modeling problem addressed in this work. Section IV presents the proposed PowerCool model. Section V presents the accuracy validation of the PowerCool model and its speed up with respect to fine-grained simulations in COMSOL. Section VI presents a case study of an MPSoC with microchannel redox flow cell array analyzed using the PowerCool model. Finally, Section VII presents the conclusions of this work.

\section{OVERVIEW OF EXISTING Modeling TeChNiQues}

Several research efforts have been made to study the activity of microfluidic flow cells, either by modeling or experimentation. Initial investigations have been made on a fabricated test structure [8]. These investigations have shown the feasibility of having such cells without a membrane, hence significantly simplifying the design process. The work by Kjeang et al. [9] has provided more insights on the microscale redox flow cell operation, when the inlet flow rates vary. In addition, they have studied the performance of a flow cell array when several electrodes are placed on a larger area. The same authors have extended their work by experimenting with porous electrodes for higher-performance microfluidic fuel cells [10].

In addition to experiments and test-vehicle fabrication, several works have targeted modeling micro-scale redox flow cells. For example, theoretical analysis of microfluidic fuel cells has been investigated in [11]. In this work, a commercial computational fluid dynamics (CFD) package (CFDRC) has been used for this analysis. Investigations on applying different flow rates, electrolytic concentrations, and microchannel geometries (i.e., aspect ratio) have been carried out. A similar work used COMSOL to examine the impact of channel and electrode geometries on microfluidic flow cell performance $[12,13]$. However these works do not consider temperaturedependent impact on flow cell performance. In addition, CFD-based models use finite-element methods, which are time consuming and do not lend themselves to be adapted to the electronic-design automation (EDA) toolchain.

Recent works have started thermal investigations on macro-scale flow cells. For example, investigations on the thermal impact on 


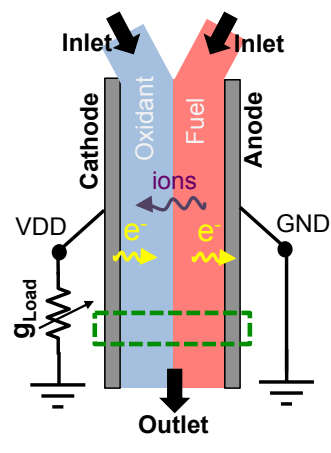

(a)

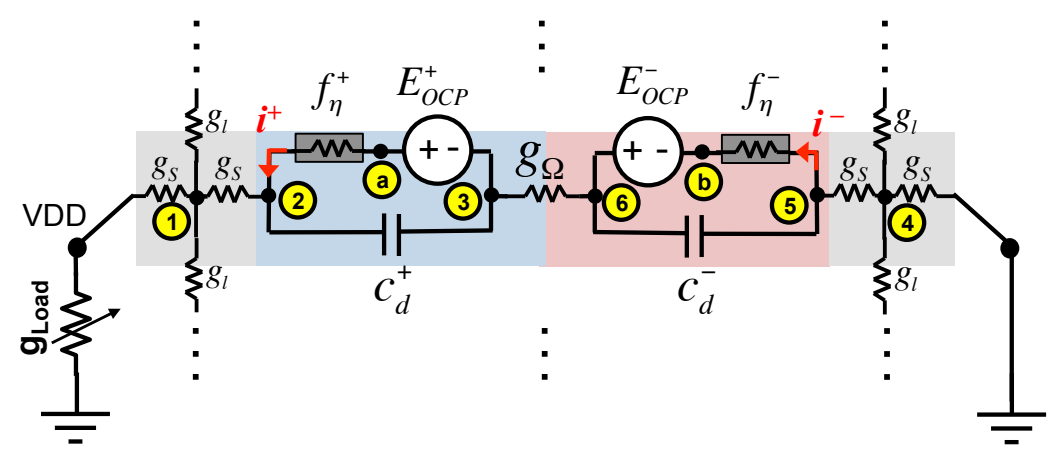

(b)

Fig. 3. (a) A single microchannel flow-cell; (b) Compact electrical model for a small section of the flow-cell.

macro-scale flow battery- a large-scale version of microfluidic flow cell- has been carried out using numerical simulations and experimental test vehicles $[14,15]$. This work has investigated the impact of increasing temperature and inlet flow rates of the fluids to maximize the battery efficiency. Another work proposes a finiteelement thermal model of flow batteries [16], where the self-heating impact of flow batteries is investigated. However, recent works on thermal investigation do not consider microfluidic flow cells. In addition, there is no investigation of the impact of external nonuniform heat sources on flow cells, large-scale or micro-scale. Indeed, the case of non-uniform heat sources is common in typical MPSoC operation [17]. These aspects are addressed in this paper.

A recent work has proposed an equivalent circuit model for microfluidic flow cells [18]. In this work an RC-circuit is introduced, which is similar to other flow cell models. The parameters used in this circuit are estimated from experimental measurements, that are run through an extended Kalman filter. The experimental measurements in this work are extracted from a macro-scale flow cell. While the main concept can be adapted to micro-scale flow cells, the model neglects numerous parameters that are essential for accuracy such as temperature, flow rate, concentration, and the electrical load.

\section{TARget Modeling Problem}

A single microchannel flow-cell with electrodes fabricated on either side- that represents a unit target structure modelled in this work- is shown in Fig. 3(a). Two species of electrolyte- one called the "oxidant" and the other "fuel"- are injected into a microchannel via separate inlets. Then, they flow in a colaminar fashion next to each other without convective mixing. Each species and its electrode together are called a single "half-cell". The oxidant absorbs electrons from its electrode and gets reduced, while the fuel gives electrons to its electrode and gets oxidized.

According to the convention, the electrode where reduction happens is called a "cathode", while the other electrode is called an "anode". In addition, the two electrolytes exchange ions with each other during this reduction-oxidation (or redox) process. Hence, if a flow-cell is used as a power source and a load is connected to the cathode as shown in Fig 3(a), the flow-cell discharges and both electrolytic species get consumed and depleted as they flow along the channel, generating an electrical current through this external circuit. It can be easily seen from the direction of the electron transfer in Fig. 3(a), that current is supplied at the cathode and returns at the anode from the external load. Hence, the cathode is essentially the VDD terminal for the IC and the anode is the ground.

For the purpose of illustration, consider a Vanadium redox flow cell, where $\mathrm{VO}_{2}{ }^{+}$is introduced as the oxidant and $\mathrm{V}^{2+}$ is introduced as the fuel. The following redox reactions take place at each half-cell:

$$
\begin{aligned}
& \mathrm{V}^{2+} \underset{\text { charge }}{\stackrel{\text { discharge }}{\rightleftharpoons}} \mathrm{V}^{3+}+\mathrm{e}^{-} \\
& \mathrm{VO}_{2}^{+}+2 \mathrm{H}^{+}+\mathrm{e}^{-} \underset{\text { charge }}{\stackrel{\text { discharge }}{\rightleftharpoons}} \mathrm{VO}^{2+}+\mathrm{H}_{2} \mathrm{O}
\end{aligned}
$$

Since we are only interested in using the flow-cell as a power source, we are only interested in the discharging direction (from left to right) of the above equations. It is impractical to elaborate on the fundamentals of electrochemical reactions and their potentials in this paper, and the readers are instead referred to $[19,20]$ for a detailed treatment of this vast subject. However, the essential concepts required to develop the compact model in this work can be summarized as follows:

1. Under zero load (zero current) conditions, each half-cell is characterized by a potential called the standard reduction potential. These half-cell potentials represent the thermodynamic equilibrium of the redox reactions described above in the direction of reduction (gain of electrons).

2. All the redox reactions and electron exchanges happen at the surface of the electrodes in the electrolyte. Bulk of the half-cell potentials resulting from these exchanges also typically appear right near the surface of the electrodes. The polarities of the anode and cathode half-cell potentials are in the same directions and hence they add together to constitute the open-circuit voltage of the flow-cell. This potential is a highly non-linear function of the surface concentrations of the redox species, as given by the "Nernst equation".

3. When a load is connected between the electrodes, current starts to flow. This causes a drop in the potential of the flow-cell. The current vs voltage relationship for the flow-cell shows highly nonlinear behavior, and is given by the "Butler-Volmer equation" for electrochemical kinetics.

4. The mobility of the ions and electrons at the electrode-electrolyte interface causes a build up of ions from the electrolyte near the electrode in each half-cell forming the electrical double layer. This acts as a capacitance during the dynamic operation of the flow-cell.

5. The ionic current between the electrolytes is subject to an ohmic resistance that depends upon the electrical properties and mobility of the different species of the redox reactions as well as the resistance of the electrodes.

\section{PowerCool: Compact Electrochemical Model FOR INTEGRATEd MiCROFluidic FuEl CELlS}

The PowerCool model uses the fundamental characteristics of electrochemical cells, described in Section III, to model the electrical behavior of the microchannel flow cells in a compact way. It must be noted the most of the theory described in Section III 
and in the literature $[19,20]$ has been described for conventional static electrochemical fuel cells/batteries where the electrolytes are stationary in fixed containers. However, these concepts can be applied to a microchannel flow cell in the PowerCool model by discretizing it into small "sections" from inlet to outlet, and assuming each section to have a constant distribution of various electrolyte species concentrations, acting as a small electrochemical fuel cell in itself. In this sense, the compact electrical model for a small section of the microchannel flow cell, highlighted using green dotted lines in Fig 3(a), is shown in Fig. 3(b).

The compact model for this small section can be divided into two symmetric parts, one for each half-cell. The various components of this compact model and its implementation are described below.

\section{A. The Nernst Voltage source}

The fundamental electrical properties of a section of the flow cell are the open circuit potentials (OCP) generated because of the redox reactions, as given by the Nernst equation (as described in Section III). This potential drop exists almost entirely near the electrode surface, and hence it is represented using a voltage source $E_{O C P}^{+/-}$ close to the electrode as shown in Fig. 3(b) (the superscripts + and - referred to the potentials at the cathode and anode respectively). Using the Nernst equation, this potential can be written for the cathode as:

$$
E_{O C P}^{+}=E_{0}^{+}-\frac{R T}{n F} \ln \frac{C_{\text {surf }}(\text { product })}{C_{\text {surf }}(\text { reactant })},
$$

where $C_{\text {surf }}$ (product) and $C_{\text {surf }}$ (reactant) refer to the concentrations of the product and the reactant species of the cathode halfcell at the electrode surface. A similar expression can be written for the anode half cell. It can be inferred from this equation that as the reactant electrolyte species get consumed in the redox equations down the channel, the fundamental half-cell potentials at both cathode and anode decrease as a logarithmic function of the ratio of the product to reactant surface concentrations.

Note: In the structure of the PowerCool model, the polarities of both $E_{O C P}^{+}$and $E_{O C P}^{-}$are shown to be in the same direction to make the intuitive understanding from a circuit perspective, that these potentials add up to form the final flow cell potential, easier. The convention in the literature of electrochemistry is to represent them with opposing polarities and writing the anode potentials $E_{O C P}^{-}$and $E_{0}^{-}$as negative quantities. In this case, the sign of the logarithm term in $\mathrm{Eq}(3)$ is also reversed.

\section{B. Butler-Volmer resistance}

The activation of the redox reactions and the subsequent mass transport of ions near the electrode surface introduces a potential loss and limits the amount of current that can be supplied by the flow cell. This process, which is highly non-linear, is characterized by an extended Butler-Volmer equation of chemical kinetics [20]. In the PowerCool model, this potential drop is modelled using a nonlinear resistor $f_{\eta}^{+/-}$(as shown in Fig. 3(b)) given by the following current-voltage relationship:

$$
i^{+/-}=f^{+/-}(\eta)=\frac{e^{\alpha \gamma \eta}-e^{-(1-\alpha) \gamma \eta}}{\frac{1}{i_{0}}+\frac{1}{i_{L}}\left\{e^{\alpha \gamma \eta}+e^{-(1-\alpha) \gamma \eta}\right\}},
$$

where $\eta$ is the potential drop across the resistor and $i_{L}=$ $n F \Delta x \Delta z D \delta^{j} \cdot C_{\text {bulk }}^{j}($ reactant $)$. Here $D$ and $\delta^{j}$ refer to the diffusion coefficient and depletion layer of the reactant species in the section (these terms are explained in Section IV-E). The direction of the current flows in the cathode and the anode branches, $i^{+}$and $i^{-}$, during the normal operation of the flow cell are indicated in Fig. 3(b).

\section{Double-layer capacitance}

The accumulation of ions near positively/negatively charge electrodes creates the Helmholtz electrical double layer that acts as a capacitance during the dynamic/transient operation of the flow cell. This capacitance acts across the same distance near the electrode surface where the half-cell potential and the Bulter-Volmer potential loss exist. Hence, it is represented as a capacitor parallel to these components in the PowerCool model as shown in Fig. 3(b). It is known for a given type of half-cell and can be obtained from the literature [20] or from experiment.

\section{Electrolyte and Electrode resistances}

The flow of ions through the bulk of the electrolytes species is subject to to an ohmic resistance represented using the conductor $g_{\Omega}$ in Fig. 3(b) between the circuits for the two half-cells. This conductance is a simple expression obtained using the electrical conductivities of the electrolytes, the dimensions of the microchannel cross-section and the section length.

In addition, the interaction between the changing potentials of the various sections along the microchannel flow cell and the electrodes is modelled by connecting the compact models for each flow cell section with a distributed resistance network representing the electrodes as shown in Fig 3(b). Here $g_{S}$ represents the electrical conduction in the lateral direction across the thickness of the electrode and $g_{l}$ represents the electrical conduction in the longitudinal direction, parallel to the microchannel. These conductances can also be obtained using simple ohm's law expressions involving the electrical conductivity of the electrode and its dimensions.

\section{E. Computation of Surface Concentrations}

As can be seen from $\operatorname{Eq}(3)$ that the computation of the voltages in the flow cell requires the knowledge of the local surface concentrations of all the four electrolytic species (one reactant and one product in each half cell) in each section. Hence, computation of these concentrations is integral to the PowerCool model. The inlet concentrations of all the four species are assumed to be known and uniformly distributed in the cross section of their respective half cell. As these species flow down the channel with current (or electrons) being drawn away from them resulting in the redox reactions, product concentrations increase at the same rate as the reactant concentrations get depleted. Assuming the concentrations within a section do not vary, the change in average concentrations of the redox species in the cathode half cell from section $j$ to the next section $j+1$ is illustrated in Fig. 4(a) and given by the following expression:

$$
\begin{aligned}
C_{\text {avg }}^{j+1}(\text { reactant })= & C_{\text {avg }}^{j}(\text { reactant })-\frac{i}{n F} \frac{\Delta z}{v} \frac{1}{\Delta x \Delta y \Delta z} \\
& =C_{\text {avg }}^{j}(\text { reactant })-\frac{i}{n F} \frac{1}{v} \frac{1}{\Delta x \Delta y} \\
C_{\text {avg }}^{j+1}(\text { product })= & C_{\text {avg }}^{j}(\text { product })+\frac{i}{n F} \frac{1}{v} \frac{1}{\Delta x \Delta y},
\end{aligned}
$$

where $\Delta z$ is the length of a section or the discretization size.

Since all of these reactions happen very close to the electrode surfaces, the distributions of these species become non-uniform, and the surface concentrations start to diverge greatly from the bulk concentrations. There is also diffusion of the reactants and products from the electrode surface into the bulk and also in the middle of the channel at the interface between the two half cells. The distribution of concentration as a result of diffusion can be given by the partial differential equations governing diffusion [21]. However, in the PowerCool model, this process can be simplified by assuming a "boundary layer" at the interfaces, where the concentrations of the species vary linearly and reach the bulk concentrations beyond it. This is illustrated for the cross-section of the $j^{\text {th }}$ section in Fig. 4(b) for all the four redox species. The boundary layer thickness $\delta$ in the $j^{\text {th }}$ section for each species can be approximated using its diffusion coefficient $D$ as [20]:

$$
\delta^{j}=\sqrt{2 D t_{j}}
$$




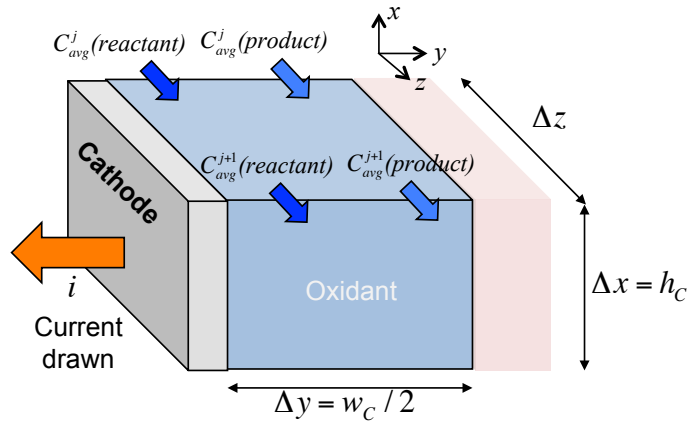

(a)
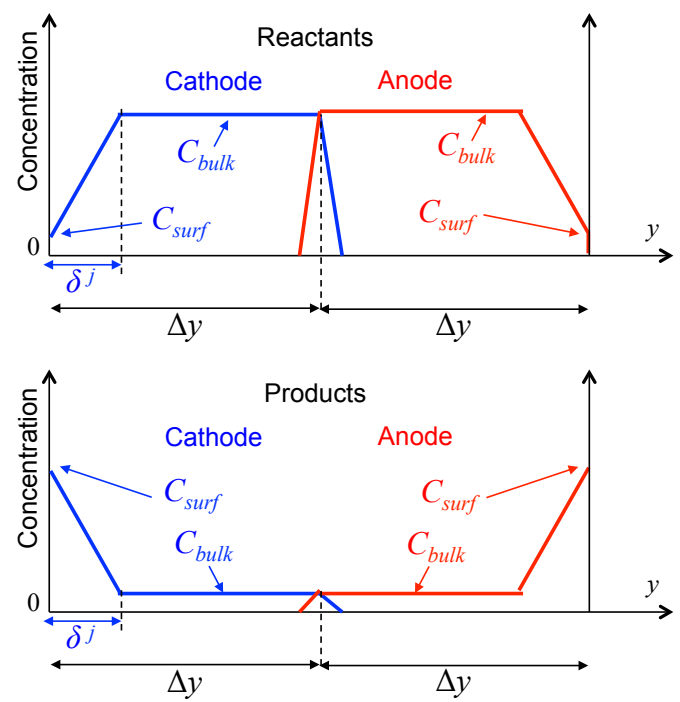

(b)

Fig. 4. (a) Change of average concentration from $j^{\text {th }}$ to $j+1^{\text {th }}$ sections; (b) Cross-sectional concentration distributions of reactants and products in the $j^{\text {th }}$ section.

where $t_{j}$ is the time the species has spent inside the microchannel since entering at the inlet and is given by $t_{j}=z_{j} / v$, where $z_{j}$ is the distance from the inlet to the $j^{\text {th }}$ section. Using simple geometrical analysis in Fig. 4(b), the surface concentrations of all reactants/products can be approximated as a function of the corresponding average concentrations computed in $\mathrm{Eq}(5)$ and (6) as follows:

$$
C_{\text {surf }}^{j}=\frac{C_{a v g}^{j} \Delta y-C_{b u l k}^{j}\left(\Delta y-\delta^{j} / 2\right)}{\delta^{j} / 2}
$$

\section{F. Implementation of the PowerCool model}

Once the circuit parameters described above are computed, modified nodal analysis can be applied to the section in Fig. 3(b) and the circuit equations can written. These individual system of equations for all the sections in a microchannel flow cell can be combined together, assuming ideal/non-ideal electrical connections between each section to the VDD/GND terminals, to write the global circuit equations for the entire microchannel flow cell in the following familiar ordinary differential equation (ODE) form:

$$
\mathbf{G X}(t)+\mathbf{C} \dot{\mathbf{X}}(t)+\mathbf{F}(\mathbf{X})=\mathbf{U}(t),
$$

where $\mathbf{X}(t)$ contains the node voltages for all the sections (there are 8 nodes in each section, indicated using numbers and letters in Fig. 3(b)) and the currents $i^{+/-}$through the Butler-Volmer resistance for all sections. $\mathbf{G}$ and $\mathbf{C}$ are the linear conductance and capacitance

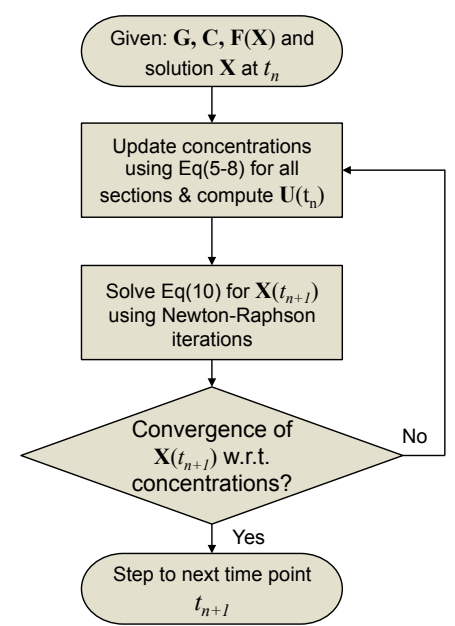

Fig. 5. Simulation flow of the PowerCool model.

matrices. $\mathbf{F}(\mathbf{X})$ contain the non-linear functions of the Butler-Volmer resistances described in $\mathrm{Eq}(4)$. $\mathbf{U}(t)$ contains the Nernst voltage sources $E_{O C P}^{+/-}$from all the sections.

The system of equations in $\mathrm{Eq}(9)$ can be numerically integrated in time using the backward Euler method to ensure numerical stability. The solution at the instant $t_{n+1}$ can then be written knowing the solution at a previous time point $t_{n}$ as follows, assuming a timestepping of $\Delta t$ :

$$
\left(\mathbf{G}+\frac{1}{\Delta t} \mathbf{C}\right) \mathbf{X}\left(t_{n+1}\right)+\mathbf{F}\left(\mathbf{X}\left(t_{n+1}\right)\right)=\mathbf{U}\left(t_{n+1}\right)+\mathbf{C X}\left(t_{n}\right) .
$$

The above system of (strongly) non-linear equations can then be solved using the Newton-Raphson method. However, it must be noted that there is a strong coupling between the concentrations of various species in the flow cell, and the currents and voltages of the model. This is because, as can be seen from from $\mathrm{Eq}(3,5-8)$, concentrations affect the Nernst voltages in each section (i.e. the $\mathbf{U}(t)$ vector in $\mathrm{Eq}(9)$ ), which affects the currents flowing through the circuits, which in turn affect the rate of electro-chemical reactions, changing the local concentrations and so on. Hence, another set of iterations (i.e. in addition to the iterations required for Newton-Raphson method) is required to compute the $\mathbf{X}$ vector at each time point. This is illustrated in the PowerCool simulation flow chart in Fig. 5.

We have developed PowerCool on MATLAB R2012b [22], which is installed on a desktop machine with Intel CORE i7-3770 CPU $3.4 \mathrm{GHz}$ and 32GB RAM. We used the inbuilt sparse matrix solver in Matlab to solve $\mathrm{Eq}(10)$. The computational complexity of the model was observed to be of the order of $O\left(N^{1.2}\right)$, where $N$ is the number of unknowns in the $\mathbf{X}$ vector.

\section{VALIDATION OF POWERCOOL MODEL}

In order to assess the efficacy of PowerCool, we examine its accuracy against a finite-element model that we have developed, as well as measurement data from the literature. To the best of our knowledge, there are no other mathematical models available in the literature for microfluidic flow cell simulation equivalent to PowerCool, that we can compare our results against. The finiteelement model was developed using the COMSOL multiphysics [23] tool, which has been used in previous works [7, 16, 24, 25]. In this section we perform the validation on two different levels, namely, single microchannel flow cell and a microchannel flow cell array. The ensuing subsections describe both validations.

\section{A. Single micro flow cell validation}

We start by validating a single microchannel flow cell [9], where vanadium redox species $\left(\mathrm{VO}_{2}{ }^{+}\right.$cathodic electrolyte, $\mathrm{V}^{2+}$ anodic 
TABLE I

LIST OF PARAMETERS $[9,26]$ USED IN THE APPLIED REDOX FLOW CELL.

\begin{tabular}{|c|c|c|c|}
\hline Parameter & Anode & Cathode & Unit \\
\hline Volumetric Flow rate & {$[2.5,10,60,300]$} & $\mu \mathrm{L} / \mathrm{min}$ \\
\hline Density & \multicolumn{2}{|c|}{1260} & $\mathrm{~kg} / \mathrm{m}^{3}$ \\
\hline Dynamic Viscosity & \multicolumn{2}{|c|}{2.53} & $\mathrm{mPa} \cdot \mathrm{s}$ \\
\hline Standard Potential $\left(E^{0}\right)$ & -0.255 & 0.991 & $\mathrm{~V}$ \\
\hline Oxidant Inlet Concentration $\left(C_{O}^{*}\right)$ & 80 & 992 & $\mathrm{~mol} / \mathrm{m}^{3}$ \\
\hline Reductant Inlet Concentration $\left(C_{R}^{*}\right)$ & 920 & 8 & $\mathrm{~mol} / \mathrm{m}^{3}$ \\
\hline Diffusion Coefficient $(D)$ & 1.7 & 1.3 & $10^{-10} \mathrm{~m}^{2} / \mathrm{s}$ \\
\hline Kinetic Rate Constant $\left(K^{0}\right)$ & 2 & 1 & $10^{-5} \mathrm{~m} / \mathrm{s}$ \\
\hline
\end{tabular}

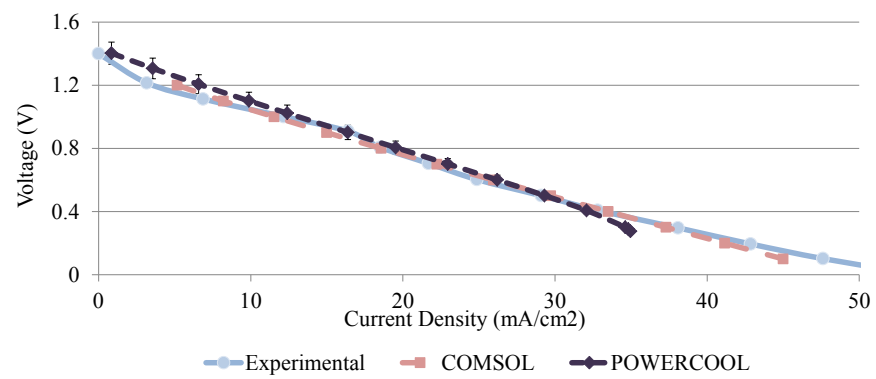

(a) $Q=300 \mu L / m i n$

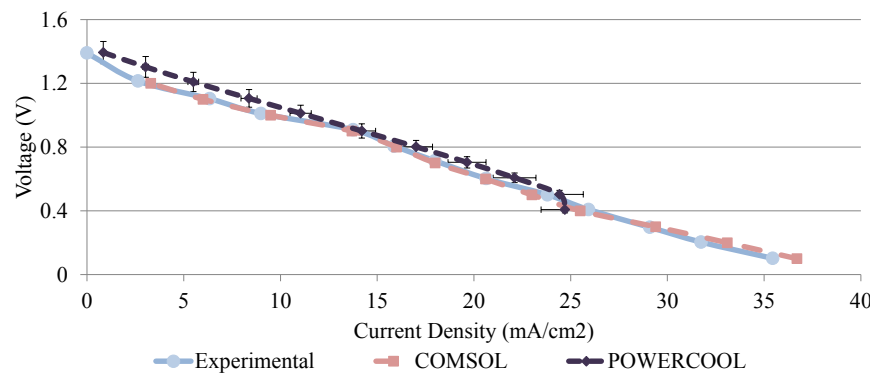

(b) $Q=60 \mu L / m i n$

Fig. 6. Validation of PowerCool accuracy with respect to COMSOL model and experimental data [9] at different flow rates. The figures have 5\% error bars surrounding PowerCool estimations to highlight its accuracy.

electrolyte) are used with graphite electrodes. We report the specific parameters we use in PowerCool and the COMSOL model to accurately simulate the flow cell in Table I. We set the discretization size in PowerCool to $100 \mu \mathrm{m}$ section length, and we set the COMSOL meshing size to extremely coarse (minimum element size is reported to be $680 \mu \mathrm{m}$ ), which is the coarsest granularity preset in COMSOL.

Figures 6(a) and 6(b) show the polarization (voltage-current density or I-V) curve of the targeted micro fuel cell when simulated with COMSOL and PowerCool. The current density here is measured as the current supplied by the flow cell per unit area of the electrode. From these figures we note the following: firstly, there is a good match between the experimental measurements, the COMSOL model, and the PowerCool. In fact, simulation results show that PowerCool incurs a maximum of 5\% error with respect to either COMSOL or the experimental data. This small error margin is accompanied by a $251 \mathrm{x}$ simulation speedup in PowerCool w.r.t. COMSOL. In early stage design explorations, $5 \%$ in voltage prediction sufficient, especially since on-chip voltage regulators, that would be placed after the flow cells, typically have a much more stringent voltage ripple/deviation specifications of $<3 \%$ [27]. The speedup is augmented to $425 \mathrm{x}$ when coarser discretization is used in PowerCool, while maintaining the same error. Secondly, there is a region where PowerCool is not functional, which occurs at high current density values. This is due to the simplified concentration diffusion model used in PowerCool (cf. Section IV-E). At high current densities, a rapid change in the surface concentrations that results in a complete "dry out"/ complete

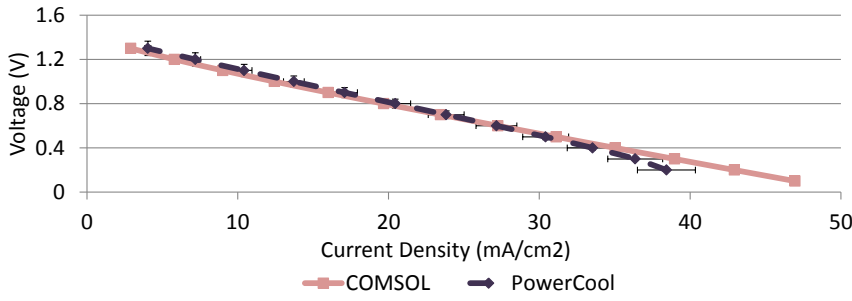

Fig. 7. Validation of PowerCool with respect to COMSOL when a microchannel flow cell array of eight microchannels is simulated. The applied flow rate is $300 \mu \mathrm{L} / \mathrm{min}$ per electrolyte.

disappearance of reactant species in the simplified PowerCool model. Fortunately, this occurs at very high current densities where the voltage levels drop to low values not encountered in design explorations. Hence, this limitation of the PowerCool model does not impact its efficacy in design.

In addition to the results shown in Figures 6(a) and 6(b), we have also examined PowerCool at the lower inlet flow rate values (i.e., $1.25,10 \mu L / \mathrm{min}$ ). At these low flow rates we observe higher errors and an even smaller coverage range of the current densities in the PowerCool model. This is also due to the low-complexity diffusion model deployed to calculate the surface concentrations. At low flow rates, the localized consumption of the redox species is increased, leading to a more complex diffusion behavior than the one described in Figure 4(b). Nevertheless, this is still acceptable for design exploration as the targeted redox flow cell array will be used for simultaneous powering-up and cooling-down the operating MPSoC which required higher flow rates (e.g. $25.2 \mathrm{~mL} / \mathrm{min}$ to meet the cooling capabilities [28])- that are orders of magnitude higher than the extreme case described above.

\section{B. Micro fuel cell array validation}

In this subsection we present the second set of validations applied on flow cell arrays, which is the typical expected design scenario where PowerCool would be used. For this, we have replicated the same single flow cell described in the previous subsection to create a flow cell array of consisting of $[2,4,8,16,32,64]$ microchannel flow cells. The geometric parameters and properties are the same as before (Table I) . The flow rate has been fixed to $300 \mu \mathrm{L} / \mathrm{min}$ per channel. These fuel cells are connected electrically in parallel (i.e. all cathodes shorted together with the VDD pin and all anodes connected to the ground pin), such that the current drawn from the array is an aggregation of all currents from the individual microchannel flow cells.

For brevity, we show the polarization curve of the 8-fuel cell array simulated by both COMSOL and PowerCool in Figure 7. This figure shows that results from PowerCool agree well with the COMSOL model. Specifically, the difference between COMSOL and PowerCool is reduced from $5 \%$ to $3.8 \%$ - i.e. a $24 \%$ reduction in error w.r.t. the simulations for a single flow cell. In addition, we see that the current density range of the PowerCool model in this case has also increased compared to the single flow cell (Figure 6(a)). This is actually a result from the increased number of fuel cells, which in turn distributes the load among the channels. Thus, the localized concentration impact on each channel is less severe compared to the case of a single channel simulation. Therefore, the simplified diffusion model adopted in PowerCool is sufficient to simulate the dynamics for flow cell arrays.

The results in Table II show the errors and speed ups of PowerCool for different flow cell array sizes when compared to the COMSOL model. These results demonstrated that PowerCool is scalable to simulate fuel cell arrays, with significant speedups (380x average speedup) and negligible error (3.8\% error). 
TABLE II

ERRORS AND SPEED-UPS OBTAINED USING POWERCOOL W.R.T COMSOL WHEN SIMULATING FUEL CELL ARRAY.

\begin{tabular}{|c|c|c|c|c|c|}
\hline Fuel cell & COMSOL Sim. & \multicolumn{2}{|c|}{$100 \mu m$ cell size } & \multicolumn{2}{|c|}{$333 \mu m$ cell size } \\
\cline { 3 - 6 } array size & Time (min:sec) & Error & Speedup & Error & Speedup \\
\hline 1 & $2: 20$ & $5 \%$ & $231 \mathrm{x}$ & $6 \%$ & $415 \mathrm{x}$ \\
\hline 2 & $3: 15$ & $5 \%$ & $235 \mathrm{x}$ & $5 \%$ & $390 \mathrm{x}$ \\
\hline 4 & $4: 48$ & $3.7 \%$ & $212 \mathrm{x}$ & $3.7 \%$ & $393 \mathrm{x}$ \\
\hline 8 & $6: 40$ & $3.8 \%$ & $160 \mathrm{x}$ & $3.8 \%$ & $423 \mathrm{x}$ \\
\hline 16 & $10: 09$ & $3.9 \%$ & $110 \mathrm{x}$ & $3.9 \%$ & $376 \mathrm{x}$ \\
\hline 32 & $20: 00$ & $3.9 \%$ & $102 \mathrm{x}$ & $3.9 \%$ & $375 \mathrm{x}$ \\
\hline 64 & $32: 30$ & $3.8 \%$ & $59 \mathrm{x}$ & $3.8 \%$ & $286 \mathrm{x}$ \\
\hline
\end{tabular}

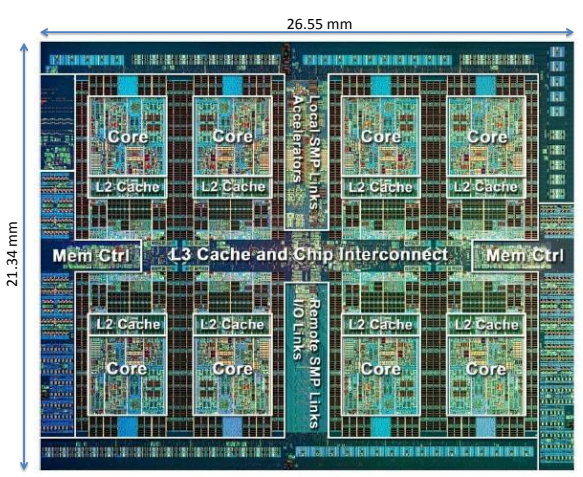

Fig. 8. Floorplan of the targeted IBM POWER7+ MPSoC.

\section{Study OF A BRIGHT-Silicon MPSOC USING POWERCOOL}

After validating the performance of PowerCool in the previous section, we will now investigate the intended purpose of integrated microfluidic power delivery and cooling using a concept MPSoC with a microchannel flow cell array. A similar case study was performed in [7] using the COMSOL tool applied to a single flow cell channel and scaling the current load to 88 channels. In this work, we apply the PowerCool model to the entire IBM POWER7+ [29] 8-core MPSoC along with an 88 channel flow cell array. This $21.34 \mathrm{~mm} \times 26.55 \mathrm{~mm}$ MPSoC (Fig. 8) has a peak power consumption density of $26.7 \mathrm{~W} / \mathrm{cm}^{2}$. A microfluidic flow cell array of 88 channels is laid out on the channel layer for this chip. In practice, the manufacturing of such structures can be realized via established microelectromechanical systems (MEMS) processes which can be highly scalable, low-cost and provide suitable materials compatibility with the chemical fuels. Since the combined cooling and power delivery fluid loops are self-contained, handling of the chemical fuels is minimal. The various structural, chemical and flow properties of this flow cell array are tabulated in Table III. To compute the cooling potential of this flow cell array, we use the 3D-ICE thermal model for liquid-cooled ICs [28], and interface it with PowerCool. This interface can also be used to study the impact of local fluid temperatures on power generated (as given in $\mathrm{Eq}(3)$ ).

For the flow cell array of 88 microchannels, the corresponding I-V curve obtained from PowerCool simulations is shown in Fig. 9. As can be seen, at a supply voltage of $1 \mathrm{~V}$, the proposed microfluidic flow cell array can provide a current slightly higher than $5 \mathrm{~A}$, which is adequate to power up the caches (L2 and L3 cache memories). The cache memories in this architecture consume an average power density of $1 \mathrm{~W} / \mathrm{cm}^{2}$, which translates to a total current requirement of $5 \mathrm{~A}$ for this chip at a supply voltage of $1 \mathrm{~V}$.

The flow cell array also serves as a microchannel heat sink. Thus, it is important to explore the cooling potential of the flow cell array and the corresponding impact on the power generation pattern. Fig. 10 shows the thermal map of the targeted MPSoC when the electrolytes are injected at the flow rate mentioned in Table III and the MPSoC is
TABLE III

LIST OF PARAMETERS [26, 30] USED IN THE MICROFLUIDIC FLOW CELL ARRAY CONNECTED TO THE IBM POWER7+ CHIP.

\begin{tabular}{|c|c|c|c|}
\hline Parameter & Anode & Cathode & Unit \\
\hline Number of channels & \multicolumn{3}{|c|}{88} \\
\hline Channel width & \multicolumn{2}{|c|}{200} & $\mu \mathrm{m}$ \\
\hline Channel height & \multicolumn{2}{|c|}{400} & $\mu \mathrm{m}$ \\
\hline Channel pitch & \multicolumn{2}{|c|}{300} & $\mu \mathrm{m}$ \\
\hline Channel length & \multicolumn{2}{|c|}{22} & $\mathrm{~mm}$ \\
\hline Volumetric flow rate (total) $\dot{V}$ & \multicolumn{2}{|c|}{676} & $\mathrm{ml} / \mathrm{min}$ \\
\hline Thermal conductivity & \multicolumn{2}{|c|}{0.67} & $\mathrm{~W} /(\mathrm{m} \cdot \mathrm{K})$ \\
\hline Thermal capacitance & \multicolumn{2}{|c|}{4.187} & $10^{6} \mathrm{~J} /\left(\mathrm{m}^{3} \cdot \mathrm{K}\right)$ \\
\hline Inlet temperature & \multicolumn{2}{|c|}{$300(27)$} & $\mathrm{K}\left({ }^{\circ} \mathrm{C}\right)$ \\
\hline Density $\rho$ & \multicolumn{2}{|c|}{1260} & $\mathrm{~kg} / \mathrm{m}^{3}$ \\
\hline Dynamic viscosity $\mu$ & \multicolumn{2}{|c|}{2.53} & $\mathrm{mPa} \cdot \mathrm{s}$ \\
\hline Standard potential $E^{0}$ & -0.255 & 1.0 & $\mathrm{~V}$ \\
\hline Oxidant inlet concentration $\left(C_{O x}^{*}\right)$ & 1 & 1000 & $\mathrm{~mol} / \mathrm{m}^{3}$ \\
\hline Reductant inlet concentration $\left(C_{R e d}^{*}\right)$ & 1000 & 1 & $\mathrm{~mol} / \mathrm{m}^{3}$ \\
\hline Diffusion coefficient $(D)$ & 4.13 & 1.26 & $10^{-10} \mathrm{~m}^{2} / \mathrm{s}$ \\
\hline Kinetic rate constant $\left(k^{0}\right)$ & 5.33 & 4.67 & $10^{-5} \mathrm{~m} / \mathrm{s}$ \\
\hline
\end{tabular}

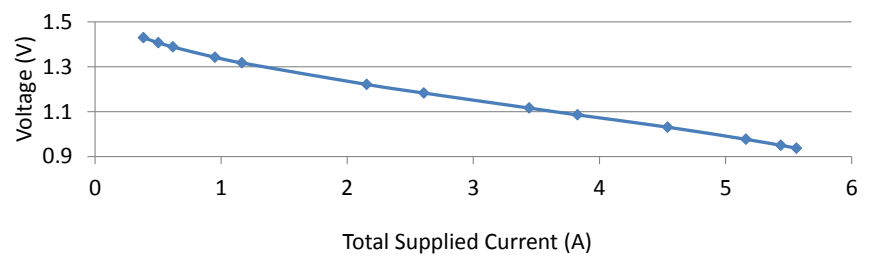

Fig. 9. Voltage-current characteristic of the microfluidic flow cell array consisting of 88 channels, simulated on PowerCool (described in Table III).

operated at full load. With the applied flow rate, which is translated to an average flow velocity of $1.4 \mathrm{~m} / \mathrm{s}$, the target MPSoC is kept at relatively low temperatures, peaking at about $41^{\circ} \mathrm{C}$. It is important to mention however that this flow rate corresponds to a pressure drop of $1.5 \mathrm{bar} / \mathrm{cm}$, which is comparable with the pressure drops used for microchannel liquid cooling in the literature [28].

To assess the energy efficiency of the target system, we calculate the pumping power needed to inject the electrolytes at the indicated flow rates (cf. Table III). Based on Darcy Weisbach pressure drop equation and Bernoulli's pumping power equation (assuming a $\eta_{p}=50 \%$ efficiency pump [31]), we find that the pumping power needed is $P=\frac{\Delta p \cdot \dot{V}}{\eta_{p}}=4.4 \mathrm{~W}$. The results in Fig. 9 demonstrate that the flow cells generate more energy than that spent in pumping, with the added benefit of providing simultaneous cooling of the entire MPSoC.

In addition to the heat removal capability of the flow cells, it is important to study the effects of the rise in flow cell temperatures in the generated power and current supplied. Electrochemical reaction rates are augmented and the power supplied generally rise with temperature. This is because of the temperature-sensitivity of several electrochemical properties such kinetic reaction rate, diffusion coefficient, electrolytic conductivity, density, dynamic viscosity, and the transfer coefficient [26]. Temperatures of a liquid-cooled IC can be increased either by increasing inlet temperature of the fluid, or by reducing the flow rates, causing slower heat-removal in the IC. Using PowerCool simulations it was found that if the flow rates are reduced to $48 \mathrm{ml} / \mathrm{min}$, or if the fluid inlet temperature is increased to $37^{\circ} \mathrm{C}$, the corresponding generated power is increased by up to $23 \%$ due to the combined enhancement of diffusion coefficient and reaction rate. The peak chip temperature under these conditions rose to only about $51{ }^{\circ} \mathrm{C}$ - still well below the $85^{\circ} \mathrm{C}$ limit for safe operation. Even the possible increase in leakage power consumption of the chip due to increased temperatures $(\sim 16 \%$ according to [32]) is outpaced by the increased performance of the electrochemical flow cell.

The latter is illustrated in terms of maximum current withdrawn from the flow cell array in Fig. 11. This observation has great 


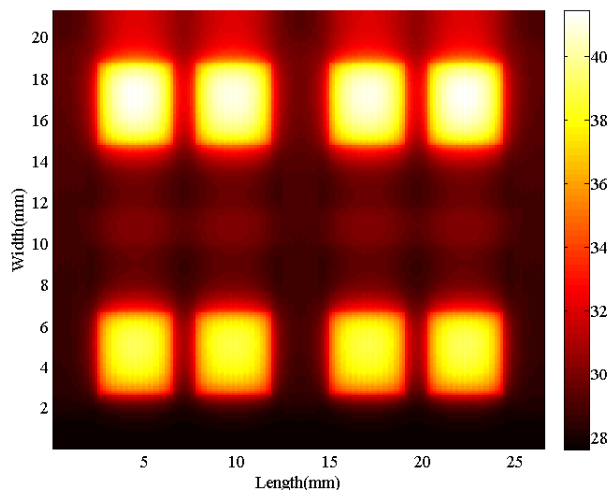

Fig. 10. Thermal response (in ${ }^{\circ} \mathrm{C}$ ) of the targeted MPSoC while cooled with a redox flow cell array.

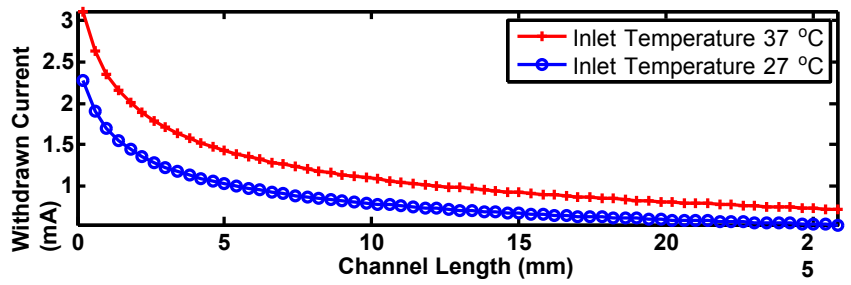

Fig. 11. Distribution of the withdrawn current from the flow cell array of 88 channels, as a function of the channel length, with different inlet flow temperatures, namely $27^{\circ} \mathrm{C}$ and $37^{\circ} \mathrm{C}$. The results shown are the average current withdrawn per channel.

significance for the design of microfluidic flow cell arrays, because it implies that high temperatures, which are normally seen as a detriment in high-performance devices, can actually be used to enable bright silicon operation of such devices. From a temperatureaware design perspective, the control of flow rates for the combined optimization of chip temperatures and pumping energy expenditure is already well known in the literature [33]. The above observation adds a potential new dimension to this early-stage design optimization for ICs with combined microfluidic power generation and cooling, and opens up a promising new research direction.

Fig. 11 also shows that the current is mainly withdrawn at the inlet, and the performance of the fuel cell degrades as we reach the output. Thus, this gives insights on how the underlying computational modules should be placed in the MPSoC layout. In particular, modules demanding higher power must be placed near the inlet, while modules requiring lower power can be placed near the outlet. This is inline with temperature-aware floorplanning observations in liquid-cooled MPSoCs [31], where high power dissipating units are preferable at the inlet while low-power dissipating modules placed at the outlet to match the degrading cooling performance of the flowing fluids along the channel length. This observation opens new research directions in localized power delivery from fuel cell arrays, in addition to examining different channel and electrode geometries for enhanced and more homogenous power generation, which is part of our future work.

\section{CONCLUSIONS}

In this paper, we presented a new compact mathematical model called PowerCool that simulates the electrical behavior of a microfluidic flow cell/flow cell array that can simultaneously powerup and cool-down an MPSoC- a technology that has been recently proposed to combat dark silicon. This model was derived from the first principles governing the behavior of voltages and currents in an electrochemical cell, and then represented as a non-linear electrical circuit that can be solved using the conventional methods for circuit simulation. PowerCool can be used to evaluate the current-voltage relationships of microchannel flow cell arrays for different loads. It's accuracy has been validated against both simulations in the finegrained COMSOL simulator as well as measurement data from the literature on this subject. Furthermore, PowerCool has been used to evaluate the recently proposed concept of bright silicon 3D MPSoC with a microchannel flow cell array demonstrating the efficacy of this model in the design of such devices and also in helping the future development of this technology.

\section{REFERENCES}

[1] H. Esmaeilzadeh et al. Dark silicon and the end of multicore scaling. In ISCA, 2011.

[2] J.H. Lau. Evolution and outlook of TSV and 3D IC/Si integration. In Electronics Packaging Technology Conference (EPTC), 2010 12th, pages 560-570, Dec 2010.

[3] N. Sturcken et al. A $2.5 \mathrm{~d}$ integrated voltage regulator using coupled-magnetic-core inductors on silicon interposer delivering $10.8 \mathrm{a} / \mathrm{mm} 2$. In $I S S C C$, pages $400-402$, Feb 2012.

[4] T. Brunschwiler et al. Interlayer cooling potential in vertically integrated packages. Microsyst. Technol., 15(1):57 - 74, 2009.

[5] P. Ruch et al. Toward five-dimensional scaling: How density improves efficiency in future computers. IBM Research and Development, 55(5):15:1-15:13, 2011.

[6] M. B. Taylor. Is dark silicon useful? Harnessing the four horsemen of the coming dark silicon apocalypse. In $D A C, 2012$

[7] M. M. Sabry et al. Integrated microfluidic power generation and cooling for bright silicon MPSoCs. In DATE 2014, pages 70-75, 2014.

[8] R. Ferrigno et al. Membraneless vanadium redox fuel cell using laminar flow. Journal of the American Chemical Society, 124(44):12930-12931, 2002

[9] E. Kjeang et al. Planar and three-dimensional microfluidic fuel cell architectures based on graphite rod electrodes. Journal of Power Sources, 168(2):379-390, 2007.

[10] E. Kjeang et al. High-performance microfluidic vanadium redox fuel cell. Electrochimica Acta, 52:4942-4946, 2007.

[11] M. H. Chang et al. Analysis of membraneless fuel cell using laminar flow in y-shaped microchannel. Journal of Power Sources, 159:810-816, 2006.

[12] A. Bazylak et al. Improved fuel utilization in microfluidic fuel cells: A computational study. Journal of Power Sources, 143:57-66, 2005

[13] A. E. Khabbazi et al. Numerical study of the effect of the channel and electrode geometry on the performance of microfluidic fuel cells. Journal of Power Sources, 195:8141-8151, 2010.

[14] B. Xiong et al. Thermal hydraulic behavior and effciency analysis of an allvanadium redox flow battery. Journal of Power Sources, 242:314-324, 2013.

[15] Z. Wei et al. Dynamic thermal-hydraulic modeling and stack flow pattern analysis for all-vanadium redox flow battery. Journal of Power Sources, 260:89-99, 2014.

[16] Q. Zheng et al. A three-dimensional model for thermal analysis in a vanadium flow battery. Appllied Energy, 113:1675-1685, 2014.

[17] J. Donald and M. Martonosi. Techniques for multicore thermal management: Classification and new exploration. In ISCA, 2006.

[18] M. R. Mohamed et al. Electrical circuit model of a vanadium redox flow battery using extended Kalman filter. Journal of Power Sources, 239:284-293, 2013.

[19] C.H. Hamann and W. Vielstich. Electrochemistry. Wiley-VCH, 2005.

[20] A.J. Bard and L.R. Faulkner. Electrochemical methods: Fundamentals and applications. John Wiley \& Sons, 2001.

[21] J. Crank. The Mathematics of Diffusion. Clarendon, Oxford, 1979.

[22] Inc. MATLAB Release 2012b, The MathWorks. http://www.mathworks.com/.

[23] COMSOL Multiphysics simulation infrastructure version 4.3.a. http://www.comsol.com/.

[24] E. Kjeang et al. Microfluidic fuel cells: A review. Journal of Power Sources, 186:353-369, 2009

[25] S. A. M. Shaegh et al. review on membraneless laminar flow-based fuel cells. International Journal of Hydrogen Energy, 36:5675-5694, 2011.

[26] T. Rapp. Electrochemical energy conversion for high power densities in microfluidic fuel cells. Master's thesis, ETH Zürich, 2012.

[27] E.A Burton et al. Fivr - fully integrated voltage regulators on 4th generation intel core socs. In Applied Power Electronics Conference and Exposition (APEC), 2014 Twenty-Ninth Annual IEEE, 2014.

[28] A. Sridhar et al. 3D-ICE: Fast compact transient thermal modeling for 3D-ICs with inter-tier liquid cooling. In ICCAD 2010, pages 463-470, 2010.

[29] IBM POWER7+. http://www-03.ibm.com/press/us/en/pressrelease/29315.wss, 2010.

[30] H. Al-Fetlawi et al. Non-isothermal modelling of the all-vanadium redox flow battery. Electrochimica Acta, 55:78-89, 2009.

[31] M. M. Sabry et al. Towards thermally-aware design of $3 \mathrm{~d}$ mpsocs with inter-tier cooling. In DATE, 2011.

[32] S. Hall and G. Kopcsay. Energy-efficient cooling of liquid-cooled electronics having temperature-dependent leakage. ASME Thermal Science and Engineering Applications, 6, 2014

[33] M. M. Sabry et al. Energy-Efficient Multi-Objective Thermal Control for LiquidCooled 3D Stacked Architectures. IEEE Trans. On CAD, 30(12), 2011. 